\title{
On the Convergence of Product Formulas for the Evaluation of Certain Two-Dimensional Cauchy Principal Value Integrals*
}

\section{By Giuseppe Mastroianni}

\begin{abstract}
The convergence of product rules of interpolatory type for the numerical evaluation of certain two-dimensional Cauchy principal value integrals is proved. Some estimates of the remainder are established for several classes of functions. Earlier results are generalized and improved.
\end{abstract}

1. Introduction. Let $\Phi(f ; s, t)$ denote the two-dimensional Cauchy principal value integral of the function $f$ defined by

$$
\begin{aligned}
\Phi(f ; s, t) & =f f_{I} \frac{f(x, y)}{(x-s)(y-t)} w_{1}(x) w_{2}(y) d x d y \\
& =\lim _{\varepsilon \rightarrow 0} \iint_{D_{\varepsilon}} \frac{f(x, y)}{(x-s)(y-t)} w_{1}(x) w_{2}(y) d x d y, \quad(s, t) \in A,
\end{aligned}
$$

where $I=[-1,1] \times[-1,1], D_{\varepsilon}=\{(x, y) \in I /|x-s| \geq \varepsilon,|y-t| \geq \varepsilon(\varepsilon>0)\}$, $w_{k} \geq 0$ and $0<\int_{-1}^{1} w_{k}(x) d x<\infty(k=1,2)$; furthermore, $A$ is the open set $(-1,1) \times(-1,1)$ with the singularities of the function $w_{1} w_{2}$ removed. We assume the existence of $\Phi(f ; s, t)$; a condition of existence can be found in the remark after Lemma 2.1 .

To approximate (1.1) we choose appropriate sets $\left\{x_{i}\right\},\left\{y_{j}\right\}$ in $[-1,1]$ and consider rules of the following type:

$$
\Phi_{m, n}(f ; s, t)=\sum_{j=1}^{n+1} \sum_{i=1}^{m+1} A_{i}(s) B_{j}(t) f\left(x_{i}, y_{j}\right),
$$

where

$$
\begin{array}{ll}
A_{i}(s)=f_{-1}^{1} \frac{l_{i}(x)}{x-s} w_{1}(x) d x, & i=1,2, \ldots, m+1, \\
B_{j}(t)=f_{-1}^{1} \frac{\bar{l}_{j}(y)}{y-t} w_{2}(y) d y, & j=1,2, \ldots, n+1
\end{array}
$$

and $l_{i}, \bar{l}_{i}$ denote the fundamental Lagrange polynomials on the knots $\left\{x_{i}\right\},\left\{y_{j}\right\}$, respectively.

The corresponding remainder $E_{m, n}(f ; s, t)$ is defined by

$$
E_{m, n}(f ; s, t)=\Phi(f ; s, t)-\Phi_{m, n}(f ; s, t) .
$$

Received July 22, 1986; revised December 19, 1986.

1980 Mathematics Subject Classification (1985 Revision). Primary 65D32, 41 A17.

*Work sponsored by the Italian Research Council. 
We observe that the rule (1.2) has degree of exactness $(m, n)$ at least, and we may obtain it using a product of interpolatory rules of type

$$
\Phi_{m}^{(1)}(g ; s)=\sum_{i=1}^{m+1} H_{i}(s) g\left(x_{i}\right)
$$

which are frequently used to approximate the one-dimensional Cauchy principal value integral

$$
\Phi^{(1)}(g ; s)=f_{-1}^{1} \frac{g(x)}{x-s} w(x) d x .
$$

Recently, the convergence of rule (1.4) has been studied in [1] under the assumption that the knots $\left\{x_{j}\right\}$ are the zeros of the orthogonal polynomials associated with a generalized smooth Jacobi weight $v(v \in \mathrm{GSJ})[5]$; that is,

$$
v(x)=\psi(x) \prod_{k=0}^{p+1}\left|s_{k}-x\right|^{\gamma_{k}}
$$

where $\gamma_{k}>-1, k \in\{0,1, \ldots, p+1\},-1=s_{0}<s_{1}<\cdots<s_{p+1}=1,0<\psi \in$ $T D:=\left\{g \in C^{0} / \int_{0}^{1} \omega(g ; u) u^{-1} d u<\infty\right\}$, with $\omega(g ; \delta)=\max _{|x-y|<\delta}|g(x)-g(y)|$, $x, y \in[-1,1], \delta \geq 0$, the modulus of continuity of the function $g$. Moreover, the authors in [1] have made the assumption that the weight $w \geq 0$ belongs to $T D$ in every closed set $X \subset B:=[-1,1]-\left\{s_{k} ; k=0, \ldots, p+1\right\}$. They have proved the following convergence result:

For any function $g \in T D$ in $[-1,1]$, if the weights $w, v$ satisfy the further conditions:

$$
w \log ^{+} w, \quad w\left(\sqrt{v \sqrt{1-x^{2}}}\right)^{-1 / 2} \text { integrable in }[-1,1],
$$

then the rule (1.4) converges uniformly to $\Phi^{(1)}(g ; s)$ on every closed set $X \subset B$. Furthermore, estimates of the remainder are established for several classes of functions.

The main object of this paper is to extend the previous convergence results to the rule (1.2). This subject has been of interest to other authors [3], [4], [6]. They proved convergence theorems in case the function $f$ is Hölder continuous and the knots $\left\{x_{i}\right\},\left\{y_{j}\right\}$ are chosen in a suitable way. We prove the convergence of rule (1.2) under weaker assumptions on the function $f$ and the weight functions $w_{1}, w_{2}$, and with more freedom in the choice of the knots $\left\{\left(x_{i}, y_{j}\right)\right\}$. The technique we use to prove our results is different from those presented in the papers mentioned above, since these latter do not seem to imply our results. We further establish estimates of the remainder which are better than those in [3], [4], [6]. This is described in Theorem 3.1, the main result of the present work.

2. Notations and Auxiliary Results. The symbol "const" stands for a positive constant taking on a different value each time it is used. It will always be clear which variables and indices the constants are independent of. Throughout this paper, $C^{k}(I)(k \geq 0)$ denotes the space of functions on $I$ that have continuous partial derivatives of all orders $i, i=0,1, \ldots, k$. If $J \subseteq I$, we set $\|f\|_{J}=\max _{J}|f(x, y)|$ and $\|f\|=\|f\|_{I}$. The modulus of continuity of functions $f \in C^{0}(J)$ is defined by

$$
\begin{array}{r}
\omega_{J}(f ; \delta)=\max \left\{\left|\Delta_{h_{1}, h_{2}} f(x, y)\right| ;(x, y) \in J,\left(x+h_{1}, y+h_{2}\right) \in J,\left|h_{1}\right|+\left|h_{2}\right|<\delta\right\}, \\
\delta \geq 0, J \subseteq I,
\end{array}
$$


where $\Delta_{h_{1}, h_{2}} f(x, y)=f\left(x+h_{1}, y+h_{2}\right)-f(x, y)$; in particular, we let $\omega(f ; \delta)=$ $\omega_{I}(f ; \delta)$. Furthermore, if $f \in C^{k}(I)(k \geq 0)$, we define the "uniform modulus of continuity" by

$$
\Omega_{k}(f ; \delta)=\max \left\{\omega\left(\partial^{k} f / \partial x^{k-i} \partial y^{i} ; \delta\right) ; i=0,1, \ldots, k\right\} .
$$

Clearly, $\Omega_{0}(f ; \delta)=\omega(f ; \delta)$. In terms of the modulus of continuity $\omega(f ; \delta)$, we define the following classes of functions:

$$
\begin{aligned}
\overline{T D} & =\left\{f \in C^{0}(I) / \int_{0}^{1} u^{-1} \log u^{-1} \omega(f ; u) d u<\infty\right\}, \\
L D(\lambda) & =\left\{f \in C^{0}(I) / \omega(f ; \delta) \log ^{\lambda} \delta^{-1}=o(1) ; \delta \rightarrow 0^{+}\right\}, \\
\operatorname{Lip}_{M} \alpha & =\left\{f \in C^{0}(I) / \omega(f ; \delta) \leq M \delta^{\alpha} ; 0<\alpha \leq 1, M>0\right\} .
\end{aligned}
$$

One can show immediately the relations

$$
L D(2) \supset \overline{T D} \supset L D(2+\beta) \supset \operatorname{Lip}_{M} \alpha, \quad \beta>0 .
$$

If we set

$$
H_{1}(g ; s, y)=\int_{-1}^{1} \frac{g(x, y)-g(s, y)}{x-s} d x, \quad H_{2}(g ; x, t)=\int_{-1}^{1} \frac{g(x, y)-g(x, t)}{y-t} d y,
$$

the following lemmas hold.

LEMMA 2.1. For any function $f \in \overline{T D}$, the inequalities

$$
\begin{aligned}
& \int_{|y-t| \leq \eta} \frac{\left|H_{1}(f ; s, y)-H_{1}(f ; s, t)\right|}{|y-t|} d y \leq 16 \int_{0}^{\eta} u^{-1} \log u^{-1} \omega(f ; u) d u \\
& \int_{|x-s| \leq \delta} \frac{\left|H_{2}(f ; x, t)-H_{2}(f ; s, t)\right|}{|x-s|} d x \leq 16 \int_{0}^{\delta} v^{-1} \log v^{-1} \omega(f ; v) d v
\end{aligned}
$$

hold uniformly on every closed set $C \subset(-1,1) \times(-1,1)$, and for $\eta \rightarrow 0^{+}, \delta \rightarrow 0^{+}$.

We remark that Lemma 2.1 and the relation

$$
\begin{aligned}
\Phi(f-f(s, t) ; s, t)= & \iint_{I} \frac{f(x, y)-f(s, t)}{(x-s)(y-t)}\left[w_{1}(x) w_{2}(y)-w_{1}(s) w_{2}(t)\right] d x d y \\
& +w_{1}(s) w_{2}(t)\left[H(f ; s, t)+\log \frac{1-s}{1+s} H_{2}(f ; s, t)\right. \\
& \left.\quad+\log \frac{1-t}{1+t} H_{1}(f ; s, t)\right],
\end{aligned}
$$

where $H=H_{1} H_{2}=H_{2} H_{1}$, imply existence and continuity of $\Phi(f ; s, t)$ in a closed set $\Lambda$, assuming that $f \in \overline{T D}$ and $w_{1} w_{2} \in \overline{T D}$ in $\Lambda$.

LEMMA 2.2. For any function $f \in C^{r}(I)(r \geq 0)$ and for any $(m, n) \in N \times N$ there exists an algebraic polynomial $P_{m, n}$ of degree at most $m$ in $x$ and $n$ in $y$ such that

$$
\begin{aligned}
\left\|f-P_{m, n}\right\| & \leq \operatorname{const}\left(m^{-1}+n^{-1}\right) \Omega_{r}\left(f ; m^{-1}+n^{-1}\right), \\
\omega\left(P_{m, n} ; \delta\right) & \leq \operatorname{const} \omega(f ; \sqrt{\delta}), \quad \delta \geq 0, \\
\omega_{C}\left(P_{m, n} ; \delta\right) & \leq \operatorname{const} \omega_{C}(f ; \delta), \quad \delta \geq 0,
\end{aligned}
$$




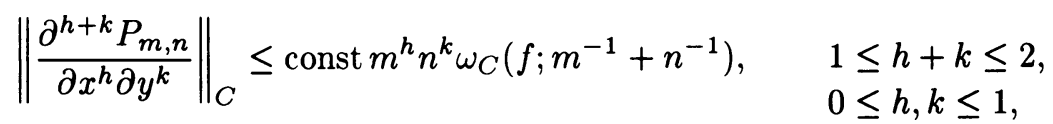

where $C$ is any closed set such that $C \subset(-1,1) \times(-1,1)$.

We point out that from (2.5), (2.6) we may obtain the relations

$$
\begin{aligned}
\omega\left(r_{m, n} ; \delta\right) & \leq \operatorname{const} \omega(f ; \sqrt{\delta}), \\
\omega_{C}\left(r_{m, n} ; \delta\right) & \leq \text { const } \omega_{C}(f ; \delta),
\end{aligned}
$$

where $r_{m, n}=f-P_{m, n}$.

LEMMA 2.3. For any function $f \in \overline{T D}$, if $w_{1} w_{2} \in \overline{T D}$ in a closed set $\Lambda \subset A$, the inequality

$$
\begin{aligned}
& \left|\Phi\left(r_{m, n}-r_{m, n}(s, t) ; s, t\right)\right| \\
& \leq \text { const }\left\{|| r_{m, n} \| \log ^{2}\left(m^{-1}+n^{-1}\right)^{-1}\right. \\
& \left.\quad+\int_{0}^{m^{-1}+n^{-1}} u^{-1} \log u^{-1} \omega(f ; u) d u\right\}
\end{aligned}
$$

holds uniformly in $\Lambda$ for $m$ and $n$ sufficiently large.

The proofs of Lemmas 2.1, 2.2, 2.3 can be found in [2]. For fixed weights $v \in$ GSJ, $w \geq 0$, let $E$ be the set of singularities of $v$ and $w$, and let $D=(-1,1)-E$. Then we have the following

LEMMA 2.4. Let $v \in$ GSJ and let $w \geq 0$ belong to $T D$ in every closed set $X \subset D$. If

$$
\begin{array}{ll}
w \log ^{+} w & \text { is integrable on }[-1,1], \\
w\left(v \sqrt{1-x^{2}}\right)^{-1 / 2} & \text { is integrable on }[-1,1]
\end{array}
$$

then the inequality

$$
\sum_{k=1}^{m+1}\left|f_{-1}^{1} \frac{l_{k}(x)}{x-s} w(x) d x\right| \leq \operatorname{const}(\log m+1)
$$

holds uniformly in $X$, where $l_{k}$ denotes the fundamental Lagrange polynomials on the zeros of the orthogonal polynomials associated with the weight $v$.

Lemma 2.4 follows immediately from a similar result which is proved in $[1$, Lemma 3.6].

3. Convergence of the Quadrature Rule $\Phi_{m, n}$. We will study the convergence of the quadrature rule $\Phi_{m, n}$ under the assumption that the knots $\left\{x_{i}\right\},\left\{y_{j}\right\}$ are the zeros of the orthogonal polynomials associated with the weights

$$
v_{1}(x)=\psi_{1}(x) \prod_{k=0}^{p+1}\left|x-s_{k}\right|^{\gamma_{k}} \in \mathrm{GSJ}, \quad v_{2}(y)=\psi_{2}(y) \prod_{l=0}^{q+1}\left|y-t_{l}\right|^{\delta_{l}} \in \mathrm{GSJ}
$$


respectively. Let $E_{i}$ be the set of singularities of $v_{i}$ and $w_{i}(i=1,2)$, and let $D_{i}=(-1,1)-E_{i}$. Let $\Delta_{i}$ be closed sets such that $\Delta_{i} \subset D_{i}(i=1,2)$. If we define $D=D_{1} \times D_{2}, \Delta=\Delta_{1} \times \Delta_{2}, \tilde{N}=\{(m, n) \in N \times N / m=O(n)\}$, then we have the following

THEOREM 3.1. Under the hypotheses

(i) $w_{i} \geq 0$ belong to $T D$ in $\Delta_{i}(i=1,2)$;

(ii) $w_{i} \log ^{+} w_{i}, w_{i}\left(v_{i} \sqrt{1-x^{2}}\right)^{-1 / 2}$ integrable in $[-1,1]$, where $v_{i} \in$ GSJ $(i=$ $1,2)$; the sequence $\left\{\Phi_{m, n}(f ; s, t)\right\}_{(m, n) \in \tilde{N}}$ converges uniformly to $\Phi(f ; s, t)$ on every closed set $\Delta \subset D$, whenever $f \in \overline{T D}$. Moreover, the relations

$$
\begin{aligned}
& \left\|E_{m, n} f\right\|_{\Delta}=o\left(\frac{1}{\log ^{\beta} n}\right), \quad f \in L D(2+\beta), \beta>0,(m, n) \in \tilde{N}, \\
& \left\|E_{m, n} f\right\|_{\Delta}=O\left(\frac{\log ^{2} n}{n^{\alpha}}\right), \quad f \in \operatorname{Lip}_{M} \alpha,(m, n) \in \tilde{N}, \\
& \left\|E_{m, n} f\right\|_{\Delta}=O\left(\Omega_{r}\left(f ; n^{-1}\right) \frac{\log ^{2} n}{n^{r}}\right), \quad f \in C^{r}(I),(m, n) \in \tilde{N},
\end{aligned}
$$

hold.

Proof. Consider the polynomial $P_{m, n}$ of Lemma 2.2. Since $\Phi_{m, n}$ has degree of exactness $(m, n)$, we have that

$$
E_{m, n}(f ; s, t)=E_{m, n}\left(r_{m, n} ; s, t\right),
$$

where $r_{m, n}=f-P_{m, n}$. Therefore,

$$
\begin{aligned}
\left|E_{m, n}(f ; s, t)\right| \leq & \left|\Phi\left(r_{m, n}-r_{m, n}(s, t) ; s, t\right)\right| \\
& +\left|\Phi_{m, n}\left(r_{m, n}-r_{m, n}(s, t) ; s, t\right)\right|=: I_{1}+I_{2} .
\end{aligned}
$$

By Lemmas 2.3, 2.4 we have

(3.6) $I_{1} \leq \mathrm{const}\left\|r_{m, n}\right\|_{\Delta} \log ^{2}\left(m^{-1}+n^{-1}\right)+\int_{0}^{m^{-1}+n^{-1}} u^{-1} \log u^{-1} \omega(f ; u) d u$,

(3.7) $I_{2} \leq 2\left\|r_{m, n}\right\|_{\Delta} \sum_{i=1}^{m+1}\left|A_{i}(s)\right| \sum_{j=1}^{n+1}\left|B_{j}(t)\right| \leq \mathrm{const}\left\|r_{m, n}\right\|_{\Delta}(\log m+1)(\log n+1)$, for $m$ and $n$ sufficiently large.

At this point we remark that, because of (2.4),

$$
\left\|r_{m, n}\right\| \leq \operatorname{const} \omega\left(f ; m^{-1}+n^{-1}\right) .
$$

Combining (3.6), (3.7), (3.8) with (3.5), we obtain

$$
\begin{aligned}
\left|E_{m, n}(f ; s, t)\right| \leq \mathrm{const} & \left\{\omega\left(f ; n^{-1}\right)\left(\log ^{2} n+1\right)\right. \\
& \left.+\int_{0}^{n^{-1}} u^{-1} \log u^{-1} \omega(f ; u) d u\right\}, \quad(m, n) \in \tilde{N},
\end{aligned}
$$

on every closed set $\Delta \subset D$ and for $n$ sufficiently large. It now suffices to observe that $L D(2) \supset \overline{T D}$ to prove the uniform convergence of $\left\{\Phi_{m, n}(f ; s, t)\right\}_{(m, n) \in \tilde{N}}$ to 
$\Phi(f ; s, t)$ on $\Delta$. Finally, from (3.6), (3.7), and by Lemma 2.3, the relations (3.1), (3.2), (3.3) follow.

We now examine some particular cases of Theorem 3.1. Let us suppose that the weights $v_{i} \in \mathrm{GSJ}, w_{i}(i=1,2)$ are such that the relations

(ii)'

$$
\begin{aligned}
& \int_{-1}^{1}\left[w_{1}(x)(1-x)^{-\max \left[\left(2 \gamma_{0}+1\right) / 4,0\right]}\right. \\
& \left.\quad \times \prod_{k=1}^{p}\left|x-s_{k}\right|^{-\max \left[\gamma_{k} / 2,0\right]}(1+x)^{-\max \left[\left(2 \gamma_{p+1}+1\right) / 4,0\right]}\right]^{r} d x<\infty \\
& \int_{-1}^{1}\left[w_{2}(y)(1-y)^{-\max \left[\left(2 \delta_{0}+1\right) / 4,0\right]}\right. \\
& \left.\times \prod_{l=1}^{q}\left|y-t_{l}\right|^{-\max \left[\delta_{l} / 2,0\right]}(1+y)^{-\max \left[\left(2 \delta_{q+1}+1\right) / 4,0\right]}\right]^{r} d y<\infty
\end{aligned}
$$

hold for some $r>1$. As the conditions (ii)' imply (ii), we obtain the following

COROLLARY 3.2. If the weights $v_{i} \in \mathrm{GSJ}, w_{i}(i=1,2)$ satisfy the assumptions (i), (ii)', then the results of Theorem 3.1 hold for the rule $\Phi_{m, n}(f ; s, t)$ and for the corresponding remainder $E_{m, n}(f ; s, t)$, whenever $f \in \overline{T D}$.

From this, we deduce

COROLlaRY 3.3. If $v_{i}(u)=(1-u)^{\alpha_{i}}(1+u)^{\beta_{i}}, \alpha_{i}, \beta_{i}>-1$, and $0 \leq w_{i} \in$ $\operatorname{Lip}_{M} \lambda$ in $\Delta_{i}, \int_{-1}^{1} w_{i}^{r}(u) d u<\infty$ for some $r>1(i=1,2)$, then the results of Theorem 3.1 hold for the rule $\Phi_{m, n}(f ; s, t)$ and for the corresponding remainder $E_{m, n}(f ; s, t)$, whenever $f \in \overline{T D}$.

This generalizes, except for the case of the practical abscissas, the results presented in [3] and [4]. On the other hand, we have the following

COROLLARY 3.4. If $w_{i}=v_{i} \in$ GSJ $(i=1,2)$, then the results of Theorem 3.1 hold for the rule $\Phi_{m, n}(f ; s, t)$ and for the corresponding remainder $E_{m, n}(f ; s, t)$, whenever $f \in \overline{T D}$.

In [6], for any function $f \in \operatorname{Lip}_{M} \alpha$, the relations

$$
\begin{aligned}
& \left\|E_{m, n} f\right\|_{\Delta}=O\left(\frac{\log ^{2} n}{n^{\alpha-\eta_{1}-\eta_{2}-1}}\right) \\
& \qquad \eta_{i}=\max \left\{\alpha_{i}, \beta_{i}\right\}>-\frac{1}{2}(i=1,2),(m, n) \in \tilde{N} \\
& \left\|E_{m, n} f\right\|_{\Delta}=O\left(\frac{\log ^{4} n}{n^{\alpha}}\right), \quad \eta_{i}<-\frac{1}{2},(m, n) \in \tilde{N},
\end{aligned}
$$

are proved, and the relation (3.2) only under the assumptions $\alpha_{i}=\beta_{i}=-\frac{1}{2}(i=$ 1,2). In (3.10), $\Delta$ denotes a closed set such that $\Delta \subset(-1,1) \times(-1,1)$. The case in which $w_{i}$ and $v_{i}$ are Jacobi weights is of interest in applications. Consequently, the following corollary is worth mentioning. 
COROllary 3.5. Let

$$
w_{i}(u)=(1-u)^{a_{i}}(1+u)^{b_{i}}, \quad v_{i}(u)=(1-u)^{\alpha_{i}}(1+u)^{\beta_{i}} \quad(i=1,2) .
$$

If

$$
\alpha_{i}<2 a_{i}+3 / 2, \quad \beta_{i}<2 b_{i}+3 / 2 \quad(i=1,2),
$$

then the results of Theorem 3.1 hold for the rule $\Phi_{m, n}(f ; s, t)$ and for the corresponding remainder $E_{m, n}(f ; s, t)$, whenever $f \in \overline{T D}$.

Dipartimento di Matematica e Applicazioni

Università di Napoli

Via Mezzocannone, 8

80134 Napoli, Italy

1. G. CRiscuolo \& G. MASTROIANNi, "On the convergence of an interpolatory product rule for evaluating Cauchy principal value integrals," Math. Comp., v. 48, 1987, pp. 725-735.

2. G. Criscuolo \& G. MAStroianni, "Convergence of Gauss type product formulas for the evaluation of two-dimensional Cauchy principal value integrals," BIT, v. 27, 1987, pp. 72-84.

3. C. DAgnino \& A. PAlamaRA ORSI, "On the evaluation of certain two-dimensional singular integrals with Cauchy kernels," Numer. Math., v. 46, 1985, pp. 121-130.

4. G. MonEGATO, "Convergence of product formulas for the numerical evaluation of certain two-dimensional Cauchy principal value integrals," Numer. Math., v. 43, 1984, pp. 161-173.

5. P. Neval, Orthogonal Polynomials, Mem. Amer. Math. Soc., no. 213, Amer. Math. Soc., Providence, R.I., 1979.

6. M. A. ŠŠKO, "Convergence of cubature processes for a two-dimensional integral," Dokl. Akad. Nauk BSSR, v. 23, 1979, pp. 293-297. 